\title{
Desiderio di realtà o realtà del desiderio?
}

L'umanità catodica in Troppi paradisi di Walter Siti

\section{Stefania Ricciardi}

\section{CpenEdition}

Journals

Édition électronique

URL : http://journals.openedition.org/cei/126

DOI : $10.4000 /$ cei. 126

ISSN : 2260-779X

Éditeur

UGA Éditions/Université Grenoble Alpes

\section{Édition imprimée}

Date de publication : 15 juin 2010

Pagination : 125-135

ISBN : 978-2-84310-168-7

ISSN : 1770-9571

\section{Référence électronique}

Stefania Ricciardi, «Desiderio di realtà o realtà del desiderio? », Cahiers d'études italiennes [En ligne],

11 | 2010, mis en ligne le 15 décembre 2011, consulté le 27 mars 2021. URL : http://

journals.openedition.org/cei/126 ; DOI : https://doi.org/10.4000/cei.126 


\title{
DESIDERIO DI REALTÀ O REALTÀ DEL DESIDERIO? \\ L'UMANITÀ CATODICA IN TROPPI PARADISI \\ DI WALTER SITI
}

\author{
Stefania Ricciardi \\ Institut supérieur de traducteurs et interprètes, Bruxelles
}

Mercoledì I3 dicembre 2006: la $R T B F$, televisione pubblica belga di lingua francese, interrompe i programmi dalle 20:30 alle 2I per un collegamento in diretta dal Palazzo Reale, dove un noto giornalista annuncia che $\mathrm{i}$ fiamminghi hanno proclamato la loro indipendenza, il re Alberto II ha lasciato il paese e il Belgio non esiste più. Questo riuscitissimo esercizio di politique-fiction, come sarà definito dal responsabile della trasmissione, ha sconvolto per mezz'ora la placida vita dei belgi. Un sondaggio ha rivelato che l' $89 \%$ della popolazione ha creduto all'istante a questa simulazione, e addirittura il $6 \%$ ha continuato a crederci persino dopo che la stessa rete, su richiesta di Fadila Laanan, ministro francofono dell'audiovisuel, precisasse a tutto schermo: "Ceci est une fiction."

Un precedente non meno eclatante, al quale gli autori del programma belga hanno confessato di essersi ispirati, risale alla colossale bufala del 30 ottobre 1938, quando un Orson Welles appena ventitreenne inscena una notte di Halloween memorabile per gli americani. Nel condurre una trasmissione radiofonica dedicata all'adattamento di un romanzo di Herbert George Wells, The War of the World, la futura celebrità annuncia che il New Jersey è stato invaso dagli extra-terrestri, seminando il panico in un milione di ascoltatori dei sei sintonizzati sulle frequenze della $C B S$ radio network.

Se l'isteria collettiva innescata da episodi del genere si smorza nel giro di pochi minuti, gli esiti più durevoli e inquietanti si registrano a freddo, quando l'artificio è stato svelato, proprio com'è accaduto a quel $6 \%$ di telespettatori belgi che rifiutavano di credere che una rete autorevole come la tivù di Stato si prestasse a un falso di cronaca, per giunta nella fascia 
oraria di massima audience, dettaglio che, al contrario, ha verosimilmente incentivato il ciak della prima politique-fiction della storia televisiva.

Perché questo tradimento? Perché far calare sui telespettatori il velo di un sospetto inaudito che va a sedimentarsi sulla percezione della realtà?

Una risposta plausibile è offerta da Troppi paradisi di Walter Siti, nato a Modena nel 1947, che tuttavia soddisfa anche ulteriori indagini, mettendo a fuoco un intrico di radici che di rado si ha la fortuna di vedere così saldamente allignate in un'opera le cui propaggini investono la sfera saggistica, antropologica, sociologica, estetica, sacro-religiosa, mediatica, linguistica e naturalmente letteraria, di una letterarietà feconda e spiazzante che tratteremo solo in rapporto al mezzo televisivo allorché meriterebbe ampi approfondimenti, come sottolineato anche dalla critica.

In questo corposo romanzo di oltre quattrocento pagine pubblicato da Einaudi nel 2006, ultimo atto della trilogia che comprende Scuola di nudo (1994) e Un dolore normale (1999), Siti s'interroga tra l'altro sull'illusione della realtà propinata nel quotidiano, nella fattispecie dalla televisione, dove il culto dell'immagine sta al telespettatore come il paradiso artificiale alla civiltà occidentale. Osserviamo anzitutto come si delineano i termini di questa proporzione pervasa da un desiderio ad ampio spettro, autentico motore del racconto, per soffermarci in seguito sulla risonanza letteraria di tale rapporto.

\section{Realtà «televisionata », immaginata e impaginata}

Il romanzo ruota intorno all'approccio, dapprima indiretto poi diretto, con la televisione da parte di un generico Walter Siti, indicato nell' $A v$ vertenza come "personaggio fittizio" in una "autobiografia di fatti non accaduti». Precisazione essenziale, dal momento che una serie di elementi - cifre onomastiche, professione, orientamento sessuale - concorrono a identificare il protagonista con l'autore, che lo fa esordire così: «Mi chiamo Walter Siti come tutti. Campione di mediocrità. Le mie reazioni sono standard, la mia diversità è di massa.» (TP, p. 3.)

$\mathrm{Al}$ di là dell' incipit rubato a Érik Satie, dichiarato nelle pagine seguenti senza tuttavia esplicitarne la fonte, spicca il desiderio d'incanalare il racconto lungo i binari dell'ordinarietà rappresentata da un personaggio che, per la sua storia fatta di passioni illecite, perversioni e nevrosi, rivendicherebbe piuttosto ascendenze da maudit. Come ha precisato l'autore nel corso della trasmissione radiofonica Fahrenheit del I7 luglio 2006, questo slittamento da una singolarità estromessa a una normalità integrata è avve- 
nuto durante gli anni della stesura, quando si è accorto che quel personaggio che stava inventando su se stesso non era il solo a covare certi desideri, per cui da emarginato rischiava di diventare una figura tipica.

Con la tivù, il Walter Siti-sosia ha un rapporto totalizzante ma tutto sommato sano, perché improntato a colmare gli spazi di solitudine e a scaricare le proprie emozioni. Finché non s'innamora di Sergio Serenelli, una persona che in televisione ci lavora, e comincia a scoprire i trucchi del mestiere e il chiacchiericcio di bassa lega tipico dell'ambiente. Dopo alcune vicissitudini amorose, Walter si lega a Marcello, culturista romano di borgata, e finisce a sua volta per lavorare come autore televisivo proprio in uno di quei programmi cosiddetti taroccati, dove vero e non vero si mescolano inestricabilmente.

Il sembrare realtà e non essere realtà costituisce il nucleo del romanzo che ha per sfondo il sistema in cui viviamo. Difatti, nel raccontare in modo smaliziato il funzionamento della televisione, Siti autore dimostra che questo meccanismo d'illusione della realtà riguarda non solo la televisione e la letteratura, ma l'intera cultura occidentale, che ha sostituito la realtà con l'immagine della realtà: «L'immagine, ecco la parola magica. Se si accettava che la realtà fosse sostituita dall' immagine della realtà, il paradiso in terra tornava ad essere possibile.» (TP, p. I34.) Si tratta di un paradiso artificiale nuovamente possibile perché «solo l'immagine può essere perfetta (e acquistabile) come la realtà mai potrebbe essere» (Siti, 2005, p. 73). L'unica realtà inamovibile è quella attestante il desiderio verso un'entità bidimensionale, priva cioè di profondità, ma capace di garantire una fruizione superficiale. Più che sostanza, l'immagine è l'apparenza, e in quanto tale viene mistificata dall'Occidente.

Siti autore è particolarmente attratto dall'universo della tivù perché il mezzo televisivo è quello che più di ogni altro si distingue per la prerogativa di trasmettere la realtà, come d'altronde sottolinea Siti personaggio: «Anche se per ragioni di budget si riempiono i palinsesti di fiction, resta che il proprio della televisione è far vedere la realtà» (TP, p. 96). A suffragio di questa idea, una ulteriore constatazione: se al cinema si vedono due persone litigare si pensa che stiano recitando mentre se la stessa scena appare in tivù si è convinti che è una lite vera (TP, p. 95).

Nel cinema, come in letteratura, l'idea di finzione è implicita: sappiamo che qualcuno, il regista o lo scrittore, ha preventivamente organizzato la materia narrata. Sebbene il rapporto tra televisione e cinema sia più stretto, perché entrambi si basano sulla potenza dell'immagine, la loro funzione risulta antitetica: «Il cinema è realizzazione onirica, la tivù è onirizzazione (cioè addormentamento) del reale» (TP, p. 96). 
Addormentare la realtà significa congelarla, sospenderla temporaneamente per servirsene all'occorrenza. Siti personaggio non contempla affatto l'idea, peraltro diffusa, che la tivù possa fungere da diversivo. A proposito dei suoi genitori, egli osserva: «A ottantadue e ottant'anni, non usano la televisione per evadere, ma per stare tenacemente aggrappati alla vita che gli sfugge» (TP, p. I5).

Trattandosi anche qui di persone comuni, che proprio come il figlio, incarnano una situazione di normalità, si potrebbe dedurre che il loro atteggiamento rispecchi quello di una precisa fascia di età. Nel prosieguo della lettura, si nota tuttavia che la richiesta di evasione è assente in maniera generica, a prescindere da fattori contingenti:

Le migliaia di persone che, nel mio palazzone o in quelli contigui, stanno guardando gli stessi programmi, non chiedono tanto di evadere quanto di ammazzare il tempo - e persino se con le cassette o il dvd stanno guardando un film, al film non chiedono la possibilità di altre vite, ma solo la licenza di anestetizzare la loro. La tivù ha televisionato anche il cinema. (TP, p. 98.)

L'impossibilità di evadere la realtà è legata al fatto che questa realtà è «televisionata», cioè manipolata, modificata, resa presentabile senza sconvolgere il sistema precostituito, come si evince da questa sequenza di frammenti:

[...] la televisione non ti fa evadere, può permettersi di essere una finestra una "finestra spalancata sul reale" perché nel frattempo il reale gli si è spiritualizzato, diventando tivù-compatibile. (TP, p. 98.)

Da quando sto con lui [Sergio] la televisione non è più (o non è più completamente) una scatola magica capace di sostituire la realtà. Ė piuttosto uno specchio deformante, che sta facendo subire alla realtà un'interessantissima torsione. [...] Dunque la realtà che passa in tivù è quella sola che si spinge fin dove i protagonisti possono osare, e che non turba gli spettatori. Quella che di solito, sbagliando, chiamiamo «irrealtà televisiva» è invece realtà depotenziata. La realtà mostrata in tivù deve essere accettabile (e produrre denaro): dunque è bene tenerla sotto controllo, aggiustarla prima che la telecamera la riprenda. La realtà televisiva è strutturata come una fiction (vedi i telegiornali, che partono dalle tragedie e finiscono nell'happy end dei divi e dello sport), ma senza avere la libertà della fiction, che è soprattutto quella di rappresentare l'estremo. (TP, pp. 95-96.)

La realtà televisiva nasce all'incrocio di due diverse potature: I) quella economica, per cui si rappresenta solo ciò che non disorienta i consumatori, cioè le masse; 2) quella psicologica, per cui l'impaginazione è a cura di individui che hanno delegato a quel lavoro la propria rassicurazione personale.

[...] La tivù non solo impagina la realtà che ti è consentito vedere, ma fa diventare «reale» quello che hai sognato al cinema, impaginandoti anche i sogni (TP, p. 97.) 
È interessante cercare di capire quando e perché scatta l'input di questo depotenziamento che trasforma la realtà in post-realtà, cioè in realtà amputata, menomata, "castrata», per ribadire un'espressione della voce narrante (TP, p. I67). In passato l'offerta finzionale era ridotta, in quanto limitata alla narrativa, serbatoio privilegiato dell'immaginazione. Anche il cinema ha operato in questa direzione, ma con una portata decisamente minore per via del sua fruizione elitaria e, fino a pochi anni fa, riservata perlopiù agli uomini, soprattutto nelle culture mediterranee. Con l'avvento della televisione, il 3 gennaio I954, si registra la prima tappa significativa di quel percorso mediatico di cui internet costituisce l'apice indiscusso. Grazie al piccolo schermo, si assiste ogni giorno alla traduzione della realtà in forma narrativa: dai fatti di cronaca, nera e rosa, alla vita politica passando per la pubblicità, il mondo che ci circonda si presenta denso di una moltitudine di dettagli che rendono tangibili persino le esperienze al di fuori della nostra portata. Inoltre, se si considera la rete di comunicazione virtuale che permette di stabilire delle connessioni con gli universi più disparati, va da sé che la presenza della fantasia nel quotidiano è diventata così elevata da forgiare a sua immagine gli atteggiamenti collettivi. Mentre in passato ci si riferiva ai genitori, agli insegnanti, agli amici, insomma, a esistenze contigue come a dei modelli di vita, oggi si tende piuttosto a imitare i divi del cinema e della televisione, le star dello sport, anche perché i media annullano le distanze senza tuttavia intaccare il mito, anzi, rafforzandolo come modello. Se internet permette un contatto con le celebrità mediante i blog, alcune trasmissioni televisive sono imperniate sull'idea di far diventare VIP una persona comune, facendo scattare nel telespettatore la molla dell'identificazione-emulazione. Si pensi ai programmi di Maria De Filippi e ai reality show che hanno coniato rispettivamente personaggi come Costantino e Pietro Taricone, quest'ultimo figura di rilievo in Troppi paradisi perché determina la rinascita del desiderio sessuale nel personaggio Walter.

Tornando al ruolo del VIP e all'evoluzione subita negli ultimi anni, oggi la Very Important Person non è, o non è solo, chi si fregia di questo titolo per ragioni dinastiche, artistiche o economiche, ma al contrario chi proviene da un contesto comune e ha acconsentito a farsi trasformare in personaggio dai media, assumendo le conseguenze che una trasformazione così repentina comporta. Il brusco passaggio dalla condizione di anonimato ad una di notorietà è illustrato magistralmente dall'episodio in cui Walter incontra, o meglio dovrebbe incontrare in privato, una VIP della televisione che finisce per disertare ogni appuntamento non per snobismo - e nel romanzo si vede bene -, ma per un banale difetto di 
comunicazione: i messaggi telefonici che i due si scambiano avvengono per interposta persona. É l'agente della VIP a sancire la crisi d'identità di un individuo a disagio nella sua nuova pelle, dove persona e personaggio si sovrappongono fino a confondersi, inducendo la VIP a farsi gestire indistintamente la sfera privata e professionale.

In questo dilagare della zona grigia, «ognuno di noi è un vip in potenza, quindi potenzialmente un'immagine» (TP, p. I4O). Un'immagine da curare con ogni artificio disponibile affinché sia impeccabile e al di fuori del tempo. Da qui il desiderio, tipico della società occidentale, per i corpi irreali scolpiti dagli anabolizzanti più che dalle palestre o ridisegnati dalla chirurgia plastica, desiderio che si estende alle griffe dell'alta moda. A differenza del passato, quando acquistare un prodotto taroccato costituiva un discrimine tra il vero e il falso, oggi le imitazioni sono apprezzate in quanto tali e non sono nemmeno spacciate per autentiche. Ciò vale anche per la chirurgia estetica, talvolta impossibile da celare, senza che questo sia fonte di pregiudizio: labbra e seni siliconati, nasi rifatti, liposuzioni sortiscono comunque il loro effetto seduttivo, perché non importa se quanto si vede è naturale o artificiale, interessa solo l'immagine che esso trasmette, le emozioni che suscita. Riaffiora in tal senso il concetto di Baudrillard della seduzione come «crime originel» che si oppone al mondo produttivo: non si tratta di fabbricare delle cose, di produrle per un mondo del valore, ma di sedurle, nell'accezione etimologica del termine, cioè di distoglierle dalla loro identità, dalla loro realtà, per votarle al gioco delle apparenze, al loro scambio simbolico (Baudrillard, 2004, pp. 27-30). Analogamente, non è un caso se nei racconti La magnifica merce, opera precedente a Troppi paradisi, compariva già la figura di Marcello, l'escort amato da Walter che, usando «il corpo come un bancomat» (TP, p. 264) rappresenta l'emblema della mercificazione.

L'universo televisivo mostra perfettamente il processo della realtà finzionalizzata. Nei programmi destinati ad un pubblico di massa, come i talk-show o i reality, ma anche nella pubblicità, sempre più spesso compare il backstage, cioè gli errori che una volta si provvedeva a tagliare in sede di registrazione. Come osserva Siti personaggio,

La televisione è l'organo respiratorio di questa fase del consumismo; distributrice di immagini (sia nel senso proprio che nel senso del clichè) con una durata e un'estensione che mai hanno avuto uguali nella storia del mondo. (TP, p. I38.)

L'esempio più eclatante di finzione che penetra nella realtà è dato dal Grande Fratello, il reality show in cui alcune persone si accingono a diventare personaggi offrendo al pubblico ogni istante della vita che conducono 
nella casa che li ospita e dalla quale non possono uscire. Benché il programma sembri concepito per catturare ogni istante di vita vera (telecamere anche a raggi infrarossi ventiquattro ore su ventiquattro), in realtà una stortura è già in atto nel copione:

[...] dovendo abolire i tempi morti già nella realtà, le regole del gioco vietano di leggere, di ascoltare la radio, di disegnare e di scrivere, di dedicarsi a quelle attività poco spettacolari che pure fanno parte del quotidiano. (TP, p. I66.)

$\mathrm{Al}$ di là di queste regole, c'è per giunta un filtro censorio che, con tagli opportuni, può far considerare non-accaduto quello che si decide di non mandare in onda. Significativo, allora, l'episodio del tentato suicidio oscurato con tale tempismo da far dubitare persino della sua esistenza, e non si può che riflettere su questa sintesi avvertita:

Insomma, la tivù ti dà l'illusione di catturare la realtà (di «superare» l'arte) proprio nel momento in cui l'ha castrata. Detto in altri termini: prima ci toglie la realtà (che non si vive perché è più comodo guardarla sul teleschermo), poi ce la regala ma riaggiustata come è utile che sia. Il tutto con un sottinteso ontologico: se si può rappresentare tutta la vita, allora la vita non è altro che ciò che si rappresenta (e un corollario: quel che non è rappresentabile in diretta tivù è semplicemente inesistente, o mostruoso). Questo ci insegna più cose, sul potere, di qualunque riflessione su Mani pulite o sul monopolio bellico degli Usa. (TP, pp. I66-167.)

La televisione vince dunque in immediatezza e forza persuasiva, perché insegna a "essere "sul tempo" " cioè a "mettere in forma la cronaca dell'anima, o delle anime, del contemporaneo che si trasforma» (TP, p. I59). Ma è anche il mezzo che certifica il desiderio con più immediatezza rispetto alla letteratura: «impari sui livelli del desiderio quel che uno scrittore bravo ci metterebbe dieci pagine a spiegarti» (TP, p. I66).

Oltre a stereotipare il desiderio, la televisione lo rende più appetibile. Non a caso, all'inizio della sua love-story con Sergio, Walter confessa: «gli occhi chiari, la barbetta rada e lo slancio della schiena nella sua eterna maglietta azzurra, be' anche per me sono più desiderabili dopo che sono stati consacrati dal video» (TP, p. 5I).

La pratica visiva finisce dunque per modalizzare la percezione della realtà. Realtà che si presenta come schermo nella duplice accezione del termine: spazio su cui si proiettano sia le forme artistiche tradotte da linguaggi diversi sia l'immaginario collettivo nelle sue espressioni comuni. Ma schermo, la realtà lo è anche in quanto maschera di un'autentificazione irreale (o, se si preferisce, di una illusione referenziale) nella misura in cui un procedimento tecnico, in particolare la diretta televisiva, permette di filmare (dunque di filtrare) un evento che si svolge in tempo reale. 
Il «crime parfait» della realtà paventato da Baudrillard nell'opera eponima del 1994 non dipenderebbe più, o almeno non solo, dallo sviluppo tecnologico (la segreteria telefonica sinonimo di una presenza irreale), ma da uno straripamento del regime finzionale. Se esiste una realtà del desiderio, è altrettanto innegabile un desiderio di realtà, sotteso nella domanda apocalittica posta da Remo Bodei: «È possibile salvare il senso della realtà dal rischio della sua dissoluzione nella fantasia?» (Bodei, p. I52.)

\section{La sospensione volontaria della credulità e le variabili dell' io}

L'iperfinzionalità del mondo stabilisce una nuova comunione d'intenti tra autore e pubblico non più basata sul patto coleridgiano, ma esattamente sul suo contrario: anziché sospendere l'incredulità, bisognerebbe preservarla. Il romanziere ostenta un rifiuto sempre più netto della fiction perché avverte ormai una autentica spossessione del mestiere: altri, con altri mezzi, non da ultimo la tivù, si sono impossessati della diffusione della finzione veicolandola spesso nelle sue forme più degradate, prime su tutte quelle della propaganda e dello sfruttamento pedagogico ossessivo.

Il processo dell'opera subisce allora un nuovo impulso: lungi dal costituire l'elemento scatenante, la fiction agisce piuttosto da garante di un metodo che eleva la scrittura al rango di opera letteraria. Come ha notato Mounir Laouyen, "Ce n'est plus le roman qui est en quête d'authenticité, c'est l'authenticité qui est en quête de fiction» (Laouyen, p. 347), idea che ha provocato uno slittamento dell'idea di verosimiglianza qual era intesa nell'Ottocento. La ricerca di questa autenticità finzionalizzata si snoda letterariamente lungo due piste spesso convergenti: quella della scrittura ibrida che attinge dalla cronaca, comunemente (e impropriamente) definita non-fiction, e quella della messinscena dell'io.

È in quest'ultima prospettiva che la trilogia di Walter Siti costituisce un fenomeno fecondo e spiazzante, si diceva prima. Fecondo, alla luce di uno sdoppiamento della prima persona autobiografica che, sulla scia di Alain Robbe-Grillet, Kenzaburo Oe e Philip Roth, annovera quel Bret Easton Ellis il cui Lunar park, per quanto deontologicamente opinabile, è esemplare per maestria tecnica. Spiazzante perché, nel suo interrogarsi sulla possibilità dell'autobiografia «al tempo della fine dell'esperienza e dell'individualità come spot», come recita l'Avvertenza, Siti scardina un sistema che né la contaminazione di matrice postmoderna né le incursioni della non-fiction nella fiction sono riuscite ad alterare. Con Troppi paradisi in particolare, egli dimostra che l'autobiografia, il genere che per antono- 
masia si inscrive nella sfera della verità, si ritrova sfrangiato nei suoi presupposti referenziali da modalità che esibiscono sin dalla denominazione palesi interferenze con la fiction. L'allusione all'autofiction è lampante, ma non è l'unica, come testimonia il Roman du Je (Forest, 2007) teorizzato e praticato da Philippe Forest.

Ma non solo. Nel suo realismo esasperante - si pensi alla minuzia dei dettagli, alle sospensioni descrittive, alla coerenza linguistica di ogni voce - Troppi paradisi mostra la perizia dell'autore nel preservare il testo da certe presenze spettacolarizzanti trattate invece come trame indispensabili di un tessuto narrativo dove nulla è gratuito. La televisione, il mondo omosessuale, l'indugiare sul desiderio e sulla pratica sessuale del protagonista, sulla sua passione smodata per un corpo perfetto quanto artefatto riflettono la fatuità della vita che Siti autore si ostina a scrivere in corsivo prima di accorgersi delle aberranti distorsioni che la investono.

La stessa oscenità ricorrente è necessaria per misurare il degrado umano del protagonista, la sua smania di un possesso mercificante che lo induce a riconoscere: «Forse è questo che sto comprando davvero: non l'intimità dei singoli escort, ma l'intimità del sistema in cui viviamo. Del suo essere una prigione in forma di paradiso artificiale» (TP, p. 2I4).

È un'oscenità eticamente necessaria, perché traduce un mondo in cui «solo il sesso può gareggiare in assolutezza con la manipolazione del reale» (TP, p. 87).

È un'oscenità che rinvia ancora a Baudrillard, a quella «visibilité totale des choses» (Baudrillard, 2004, p. 36), a quella «transparence [...] meurtrière» (idem) che, di fatto, ha finito per uccidere la vena creativa del personaggio diventato autore televisivo:

Certe volte, mentre sto per mettermi al computer e confezionare una storia, mi assale lo scoramento e preferirei sbattere la testa contro il muro. Sto distruggendo con le mie mani quel che ho di più caro al mondo: l'arte, quella vera - quella in cui si entra come in religione. (TP, p. 335.)

Anche l'autobiografia è morta. La forma narrativa si rinnova insieme a un personaggio che, dopo più di mille pagine, nasce, e si presenta alla vita con una nuova consapevolezza che ha spento il desiderio di narrarsi, ma non quello di narrare. Difatti il romanzo si chiude su un'espressione al futuro, di quelle che in un manuale di grammatica potrebbe fornire un esempio di "periodo ipotetico di primo tipo: ipotesi realizzabile»: «se avrò qualcosa da raccontare, non sarà su di me» (TP, p. 425).

L'uomo partorito dalla trilogia, autentica «formazione al nascere» (Casadei, 2007, p. 248), non sarà più personaggio, ma solo autore. Un 
autore che, come ha scritto Gianluca Nicoletti, ha tratto «l'ispirazione per la sua ricerca di umanità residue nel più disumano degli universi»" (Nicoletti, 2007) sconvolgendo almeno due sistemi precostituiti. Sfiduciati i mezzi più credibili per antonomasia, la televisione e l'autobiografia, Walter Siti-come-tutti deve attingere altrove i parametri di un nuovo codice da configurare nella propria umanità catodica, cioè in quella parte di sé che, proprio come il vecchio tubo semiestinto, si adopera per ricostruire immagini visibili di una realtà sempre più schermata.

\section{Bibliografia}

\section{Opere (narrativa e critica)}

Baudrillard Jean, Le Crime parfait, Paris, Galilée, I994.

-, Mots de passe, Paris, Le Livre de Poche, [2000] 2004.

Casader Alberto (a cura di), Spazi e confini del romanzo. Narrative tra Novecento e Duemila, Bologna, Pendragon, 2002.

- Stile e tradizione nel romanzo italiano contemporaneo, Bologna, Il Mulino, 2007.

Ellis Bret Easton, Lunar park, trad. it., Torino, Einaudi, 2005.

Forest Philippe, Le Roman, le réel et autres essais, Nantes, Cécile Defaut, 2007.

Hamburger Käte, Logique des genres littéraires, Paris, Seuil, [1957] 1986. SatıE Érik, Écrits, réunis, établis et annotés par Ornella Volta, Paris, Éd.

Champ Libre, 1970 (nouvelle édition revue et augmentée 198I).

Siti Walter, Scuola di nudo, Torino, Einaudi, 1994.

-, Un dolore normale, Torino, Einaudi, 1999.

—, La magnifica merce, Torino, Einaudi, 2004.

—, Troppi paradisi, Torino, Einaudi, 2005.

\section{Articoli apparsi in volumi collettivi elo riviste}

Boder Remo, "Vite parallele. Etica e romanzo», in Spazi e confini del romanzo. Narrative tra Novecento e Duemila, a cura di Alberto Casadei, Bologna, Pendragon, 2002, pp. I5I-I6I.

ChoukHadarian Govanni, «Se il mondo non sta nel romanzo», L'Indice, n. IO, ottobre 2006.

Lagioia Nicola, «Italia, televisione, mutazione: un grande romanzo di Walter Siti», Lo straniero, n. 76, ottobre 2006.

LAOUyen Mounir, «L'autofiction : une réception problématique», in Alexandre Gefen e René Audet (dir.), Frontières de la fiction, Éditions 
Nota bene (Québec) \& Presses universitaires de Bordeaux, 200I, pp. 339-355.

Nicoletri Gianluca, «Il Professore e la TV spazzatura», La Stampa, I7 luglio 2006.

Siti Walter, «Il 'recitar vivendo' del 'talk-show' televisivo», in Contemporanea, III, pp. 73-78. 
